\title{
Luminescence tuning and enhanced nonlinear optical properties of nanocomposites of $\mathrm{ZnO}-\mathrm{TiO}_{2}$
}

\author{
Litty Irimpan $^{\mathrm{a}, *}{ }^{\text {, Bindu Krishnan }}{ }^{\mathrm{b}}$, V.P.N. Nampoori ${ }^{\mathrm{a}}$, P. Radhakrishnan ${ }^{\mathrm{a}}$ \\ a International School of Photonics, Cochin University of Science and Technology, Cochin, Kerala, India \\ b Centre for Materials for Electronics Technology, Thrissur, India
}

\section{A R T I C L E I}

\section{Article history:}

Received 26 February 2008

Accepted 24 April 2008

Available online 29 April 2008

\section{Keywords:}

Nanocomposites

Fluorescence emission

Nonlinear absorption coefficient

Two photon absorption and nonlinear

refractive index

\begin{abstract}
A B S T R A C T
In this article we present the spectral and nonlinear optical properties of $\mathrm{ZnO}^{-\mathrm{TiO}_{2}}$ nanocomposites prepared by colloidal chemical synthesis. Emission peaks of $\mathrm{ZnO}_{-} \mathrm{TiO}_{2}$ nanocomposites change from $340 \mathrm{~nm}$ to $385 \mathrm{~nm}$ almost in proportion to changes in $E_{\mathrm{g}}$. The nanocomposites show self-defocusing nonlinearity and good nonlinear absorption behaviour. The nonlinear refractive index and the nonlinear absorption increase with increasing $\mathrm{TiO}_{2}$ volume fraction at $532 \mathrm{~nm}$ and can be attributed to the enhancement of exciton oscillator strength. $\mathrm{ZnO}-\mathrm{TiO}_{2}$ is a potential nanocomposite material for the tunable light emission and for the development of nonlinear optical devices with a relatively small limiting threshold.
\end{abstract}

(c) 2008 Elsevier Inc. All rights reserved.

\section{Introduction}

Semiconductor nanoparticles have been under continuous scientific interest because of their unique quantum nature, which changes the material solid-state properties. The linear and nonlinear optical properties of semiconductors are the subject of much current theoretical and experimental interest [1]. Amongst the various nonlinear optical (NLO) materials investigated, direct bandgap semiconductors, especially zinc oxide $(\mathrm{ZnO})$ and titanium dioxide $\left(\mathrm{TiO}_{2}\right)$ have attractive nonlinear properties that make them ideal candidates for NLO based devices. ZnO is a wide and direct band gap II-VI semiconductor with a band gap of $3.37 \mathrm{eV}$ and a high exciton binding energy of $60 \mathrm{meV}$ having many applications, such as a transparent conductive contact, thin-film gas sensor, varistor, solar cell, luminescent material, surface electroacoustic wave device, heterojunction laser diode, UV laser, and others. Nanosized $\mathrm{ZnO}$ in the form of quantum dots, nano wires, nano belts, etc., are referred to as the material of the 21st century [2]. The optical properties of this material are currently the subject of tremendous investigations, in response to the industrial demand for optoelectronic devices that could operate at short wavelengths. There is a significant demand for high nonlinear optical materials, which can be integrated into an optoelectronic device.

In recent years, interest in the synthesis, characterization, and application of colloidal "quantum dot" semiconductor materials has grown markedly [3]. Nanoscale composite materials containing ti-

\footnotetext{
* Corresponding author.

E-mail address: littyirimpan@yahoo.co.in (L. Irimpan).
}

tanium oxides are interesting because of their potential applications in optoelectronic devices and the bulk $\mathrm{TiO}_{2}$ has a direct band gap of $3.2 \mathrm{eV}$ [4]. A great deal of research effort has been focused on both synthesis of $\mathrm{TiO}_{2}$ nanocomposites, and on their linear optical properties. Recently the nonlinear optical properties of such materials have also received attention. A large, reverse saturable type of nonlinear absorption is observed in polystyrene maleic anhydride- $\mathrm{TiO}_{2}$ nanocomposites with a continuous wave $\mathrm{He}-\mathrm{Ne}$ laser beam [5]. Here, we report our investigation on spectral and nonlinear optical characteristics of $\mathrm{ZnO}-\mathrm{TiO}_{2}$ nanocomposites. Our results show that such nanocomposites possess bandgap engineering, fluorescence tuning, very large optical nonlinearity and have a great potential for optical switching and optical communications.

The possibility of tailoring the bulk material properties by varying the size, structure, and composition of constituting nanoscale particles makes them candidates for various important applications in the field of material research. The field of nanocomposite materials has been widely recognized as one of the most promising and rapidly emerging research areas. Promising applications are expected or have already been realized in many fields of technology such as optical and electronic materials, solid electrolytes, coating technology, sensorics, catalysis, and separation science [6]. Such composite materials are especially of interest in developing efficient light-energy conversion systems, optical devices, and microelectronics [7].

With many advantages such as low cost, nontoxicity, and stability, ZnO is becoming a very promising $n$-type oxide semiconductor. Most of the work has been devoted to the electrical and fluorescent properties of ion-doped zinc oxide materials [8], while only a few reports can be found using $\mathrm{ZnO}$ as the matrix for 
nanoparticle composite films [9]. These nanocomposites may lead to optically functional properties. Extensive investigations of the photoluminescence and the third order optical nonlinearities of nanometer-sized semiconductor materials have demonstrated interesting physical properties and potential applications. The absorption and luminescent properties of $\mathrm{TiO}_{2}, \mathrm{CdS}$ and $\mathrm{PbS}$ particles can be easily tuned by selecting appropriate matrix materials [10]. Recently, a microemulsion technique has been developed to prepare semiconductor nanocomposites such as $\mathrm{ZnS} / \mathrm{CdSe}$, ZnSe/CdSe, $\mathrm{ZnS} / \mathrm{CdS}$ or $\mathrm{TiO}_{2}-\mathrm{SiO}_{2}$ in a core-shell structure [11]. Chemically synthesized semiconductor nanocomposites offer necessary and basic materials promising color-tunable, flexible, all-purpose chromophore systems, in which the strong quantum confinement effect of the carriers leads to unique, sizedependent linear and nonlinear optical properties [12]. In this study, therefore, the nanocomposite techniques are applied to improve the spectral and optical properties of $\mathrm{ZnO}$. In our continued efforts to explore the optical properties of various nanocomposites, we have now elucidated the spectral and nonlinear response $\mathrm{ZnO}-\mathrm{TiO}_{2}$ nanocomposites.

Generally, photoluminescence (PL) spectrum of a single crystal $\mathrm{ZnO}$ consists mainly of two bands [13]. The one in the UV region corresponding to the near band edge emission at about $380 \mathrm{~nm}$ is mainly attributed to near band edge emission, and the other in the visible region is due to structural defects and impurities. Soon after the reporting of stimulated UV emission of $\mathrm{ZnO}$ at room temperature, it attracted the attention of the researchers as a UV laser material [14]. Thereafter, more and more investigators aimed at applications of $\mathrm{ZnO}$ emitting at the short wavelength. Several reviews elaborated the recent development of photoelectron applications of $\mathrm{ZnO}$ in short wavelength [15]. Tsukazaki et al. reported the violet electroluminescence from homostructural $\mathrm{ZnO} p-i-n$ junctions at room temperature [16]. However, the improvement of UV emission and the simplification of growth techniques are still very important.

We report the wavelength dependendant nonlinear absorption of $\mathrm{ZnO}-\mathrm{TiO}_{2}$ nanocomposites with varying $\mathrm{TiO}_{2}$ content under nanosecond excitation. The studies of nonlinear processes in photonic materials are significant in the context of their technological applications, especially in areas such as passive optical power limiting, optical switching, and the design of logic gates. Optical limiting occurs when the absolute transmittance of a material decreases with increase in input fluence. One mechanism for optical limiting is provided by RSA, in which the excited state absorption cross section is higher than the ground state absorption cross section. It is also known that doping significantly improve the limiting performance of $\mathrm{ZnO}$.

\section{Experimental}

Colloids of $\mathrm{ZnO}$ are synthesized by a modified polyol precipitation method [17]. The monodisperse ZnO colloidal spheres are produced by a two-stage reaction process. The method of preparation involves the hydrolysis of zinc acetate dihydrate $(\mathrm{ZnAc})$ in diethylene glycol medium (DEG). Among the different polyols, diethylene glycol (DEG) is chosen because it is reported to give particles with uniform shape and size distribution. The size of the particles and hence the stability of this colloidal suspension depends on the concentration of zinc acetate as well as on its rate of heating. The molar concentration of precursor solution is $0.025 \mathrm{M}$ and a heating rate of $4{ }^{\circ} \mathrm{C}$ per minute is employed for the formation of $\mathrm{ZnO}$ at a temperature of $120^{\circ} \mathrm{C}$. The product from the primary reaction is placed in a centrifuge and the supernatant (DEG, dissolved reaction products, and unreacted $\mathrm{ZnAc}$ and water) is decanted off and saved. A secondary reaction is then performed to produce the monodisperse $\mathrm{ZnO}$ spheres. Prior to reaching the working temperature, typically at $115^{\circ} \mathrm{C}$, some volume of the primary reaction supernatant is added to the solution. After reaching $120^{\circ} \mathrm{C}$, it is stirred for one hour, to get a monodisperse stable colloid.

The $\mathrm{TiO}_{2}$ nanocolloids are prepared by hydrolysis method [18]. Titanium tetrabutoxide (Aldrich) is used as the precursor. In a typical synthesis, $0.025 \mathrm{M}$ titanium tetrabutoxide is dispersed in distilled water under stirring. The solution is kept on stirring for one hour to get a monodisperse stable colloid.

The $\mathrm{ZnO}-\mathrm{TiO}_{2}$ nanocomposites are prepared by colloidal chemical synthesis by mixing certain amount of $\mathrm{TiO}_{2}$ colloid to $\mathrm{ZnO}$ colloid and stirred for $1 \mathrm{~h}$. The volume fraction of $\mathrm{TiO}_{2}$ is changed keeping the volume of $\mathrm{ZnO}$ constant. The samples having $\mathrm{ZnO}$ $x \mathrm{TiO}_{2}$ composition with $(x=) 0.1 \%, 0.5 \%, 1 \%, 2 \%, 5 \%$ are named as $\mathrm{ZnO}-0.1 \mathrm{TiO}_{2}, \mathrm{ZnO}-0.5 \mathrm{TiO}_{2}, \mathrm{ZnO}-1 \mathrm{TiO}_{2}, \mathrm{ZnO}-2 \mathrm{TiO}_{2}$ and $\mathrm{ZnO}-5 \mathrm{TiO}_{2}$, respectively.

The $\mathrm{ZnO}-\mathrm{TiO}_{2}$ nanocomposites are characterized by optical absorption measurements recorded using a spectrophotometer (JascoV-570 UV/vis/IR) and the fluorescence emission measurements are recorded using a Cary Eclipse fluorescence spectrophotometer (VARIAN). In the present investigation, we have employed the single beam $z$ scan technique with nanosecond laser pulses to measure the nonlinear optical absorptive and refractive properties of $\mathrm{ZnO}-\mathrm{TiO}_{2}$ nanocomposites. $z$ scan technique developed by Sheik Bahae and his co-workers is a single beam method for measuring the sign and magnitude of nonlinear refractive index, $n_{2}$, and has a sensitivity comparable to interferometric methods $[19,20]$. A Q-switched Nd:YAG laser (Spectra Physics LAB-1760, $532 \mathrm{~nm}$, $7 \mathrm{~ns}, 10 \mathrm{~Hz}$ ) is used as the light source. The sample is moved in the direction of light incidence near the focal spot of the lens with a focal length of $200 \mathrm{~mm}$. The radius of the beam waist $\omega_{0}$ is calculated to be $35.4 \mu \mathrm{m}$. The Rayleigh length, $z_{0}=\left(\pi w_{0}^{2}\right) / \lambda$ is estimated to be $7.4 \mathrm{~mm}$, much greater than the thickness of the sample cuvette $(1 \mathrm{~mm})$, which is an essential prerequisite for $z$ scan experiments. The transmitted beam energy, reference beam energy and their ratio are measured simultaneously by an energy ratiometer (Rj7620, Laser Probe Corp.) having two identical pyroelectric detector heads (Rjp735). The linear transmittance of the far field aperture $S$, defined as the ratio of the pulse energy passing the aperture to the total energy is measured to be approximately 0.21 . The $z$ scan system is calibrated using $\mathrm{CS}_{2}$ as the standard. The effect of fluctuations of laser power is eliminated by dividing the transmitted power by the power obtained at the reference detector. The data are analyzed by using the procedure described by Sheik Bahae et al. and the nonlinear coefficients are obtained by fitting the experimental $z$ scan plot with the theoretical plots.

\section{Results and discussion}

Optical absorption measurement is an initial step to observe the single colloid and nanocomposite behaviour. Fig. 1 gives the room temperature absorption spectra of the $\mathrm{ZnO}^{-\mathrm{TiO}_{2}}$ nanocomposites. The excitonic peak of $\mathrm{ZnO}$ and that of $\mathrm{TiO}_{2}$ colloids are found to be blue shifted with respect to their bulk which could be attributed to the confinement effects. The pronounced dependence of the absorption band edge on the size of semiconductor nanocrystals is used to determine the particle size. An order of magnitude estimate of the particle size is possible from the absorption spectra. From the shift of absorption edge, size of the dots is calculated. The size of $\mathrm{ZnO}$ and $\mathrm{TiO}_{2}$ nanocolloids are in the range of $8-10 \mathrm{~nm}$. The presence of excitonic peak itself indicates that the composites are of nanometer size. It is seen that the absorption edge corresponding to the nanocomposites gets red shifted as a function of the $\mathrm{TiO}_{2}$ content compared to pure $\mathrm{TiO}_{2}$. The size evolution of nanocomposites may also have some relation with optical characteristics in addition to the composition and the study is in progress. 


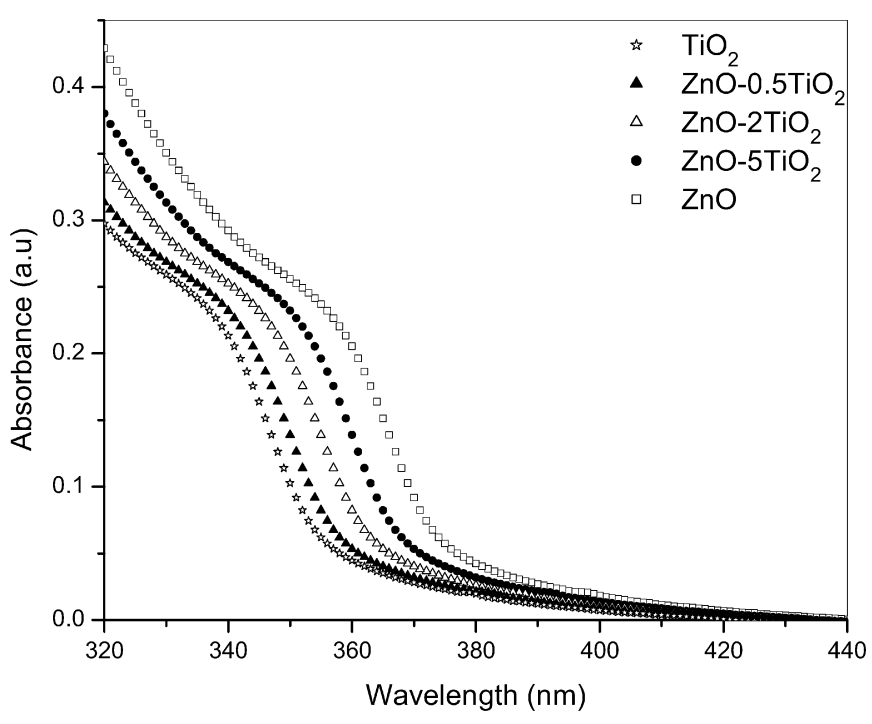

Fig. 1. Absorption spectra of $\mathrm{ZnO}-\mathrm{TiO}_{2}$ nanocomposites.

The direct bandgap of $\mathrm{ZnO}-\mathrm{TiO}_{2}$ nanocomposites is estimated from the graph of $h v$ versus $(\alpha h v)^{2}$ for the absorption coefficient $\alpha$ that is related to the bandgap $E_{\mathrm{g}}$ as $(\alpha h v)^{2}=k\left(h v-E_{\mathrm{g}}\right)$, where $h v$ is the incident light energy and $k$ is a constant. The optical band gap $\left(E_{\mathrm{g}}\right)$ is found to be dependent on the composition and there is a decrease in the band gap of the semiconductor with an increase in volume fraction of $\mathrm{TiO}_{2}$ in the nanocomposites compared to pure $\mathrm{TiO}_{2}$ as shown in Fig. 2. This is because higher carrier concentration in conduction and valence bands lead to bandgap reduction due to enhanced carrier-carrier interaction as well as by the distortion of the crystal lattice since the optical properties of the nanocolloids strongly depend on the micro-structure of the materials. Both bandgap narrowing mechanisms add up and they are often hard to separate [21]. Eg changes from 3.84 to $4.12 \mathrm{eV}$ almost in proportion to the composition of $\mathrm{TiO}_{2}$. The total change in the band gap of the material is contributed by the shifts of the valence band as well as that of the conduction band edges away from each other. In general, the shift of the top of the valence band (TVB) is not the same as that of the bottom of the conduction band $(\mathrm{BCB})$. Moreover, there are recent studies, though few in number that report the individual shifts in TVB and BCB employing various forms of high-energy spectroscopies, such as the photoemission and the X-ray absorption spectroscopies [22]. The shifts of the band edges decrease smoothly to zero for large sized nanocrystals and the shift in the BCB is in general much larger compared to the shift in the TVB. Within the range of compositions studied, the optical band gap is tunable from 3.84 to $4.12 \mathrm{eV}$. The bandgap engineering in $\mathrm{ZnS}-\mathrm{CdS}$ is reported to be from 2.58 to $3.91 \mathrm{eV}$ [23].

Photoluminescence spectra of all samples for an excitation wavelength of $300 \mathrm{~nm}$ measured at room temperature are shown in Fig. 3. The $385 \mathrm{~nm}$ emission is the near band edge emission of $\mathrm{ZnO}$ and the $340 \mathrm{~nm}$ emission is the near band edge emission peak of $\mathrm{TiO}_{2}$. Emission peaks of $\mathrm{ZnO}-\mathrm{TiO}_{2}$ nanocomposites changes from $340 \mathrm{~nm}$ to $385 \mathrm{~nm}$ almost in proportion to changes in $E_{\mathrm{g}}$. It is possible to obtain a desired wavelength of UV luminescence by simply adjusting the composition. The tuning of luminescence in $\mathrm{ZnS}-\mathrm{CdS}$ is reported to be from blue to red in proportion to change in $E_{\mathrm{g}}[23]$.

Fig. 4 shows the nonlinear absorption of $\mathrm{ZnO}-\mathrm{TiO}_{2}$ nanocomposites at a typical fluence of $300 \mathrm{MW} / \mathrm{cm}^{2}$ for an irradiation wavelength of $532 \mathrm{~nm}$. The open-aperture curve exhibits a normalized transmittance valley, indicating the presence of induced absorption in the colloids. The obtained nonlinearity is found to

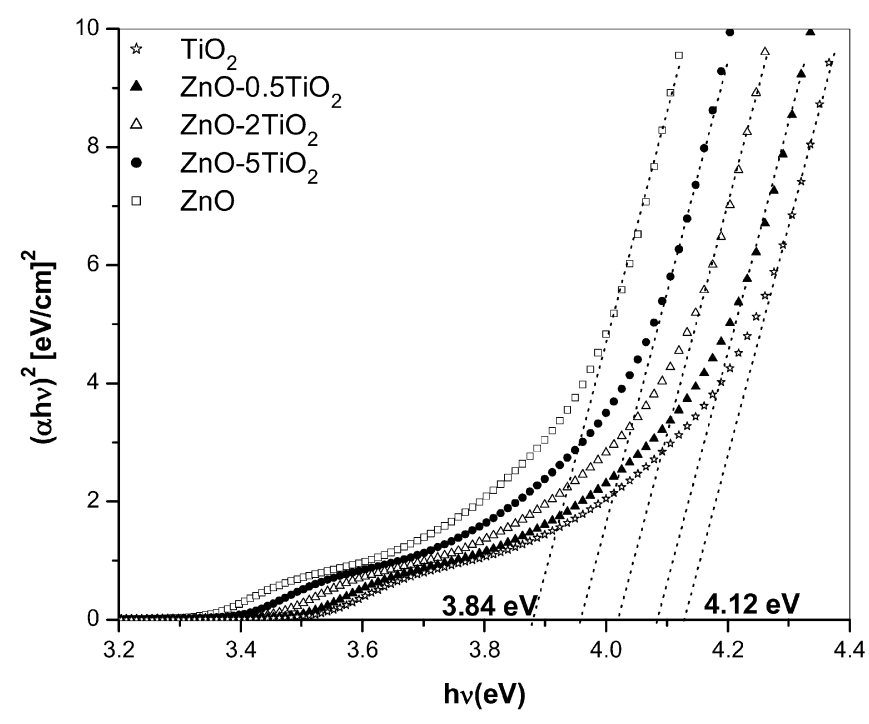

Fig. 2. Optical band gap of $\mathrm{ZnO}-\mathrm{TiO}_{2}$ nanocomposites.

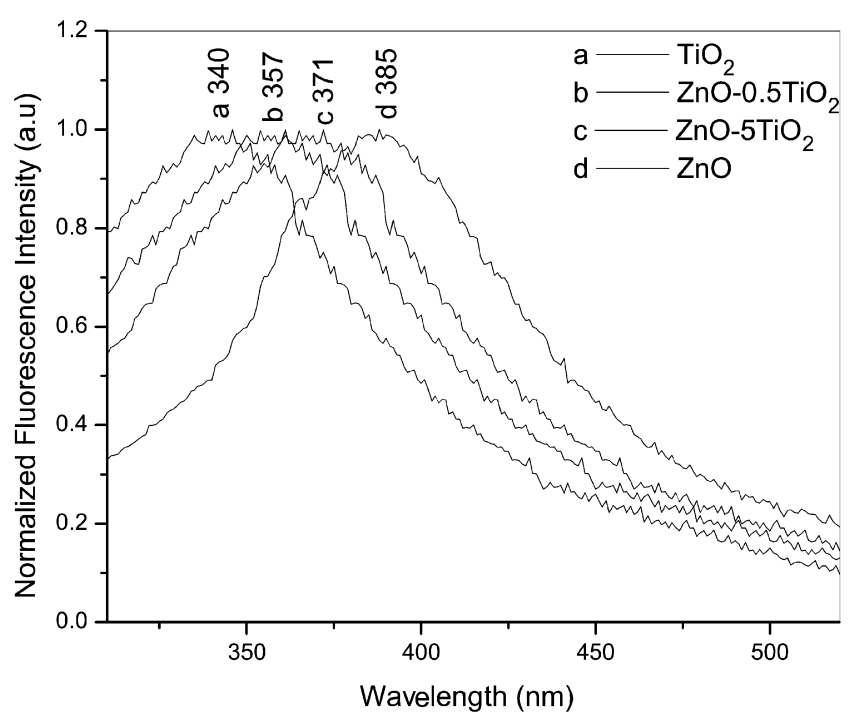

Fig. 3. Fluorescence spectra of $\mathrm{ZnO}-\mathrm{TiO}_{2}$ nanocomposites for an excitation wavelength of $300 \mathrm{~nm}$.

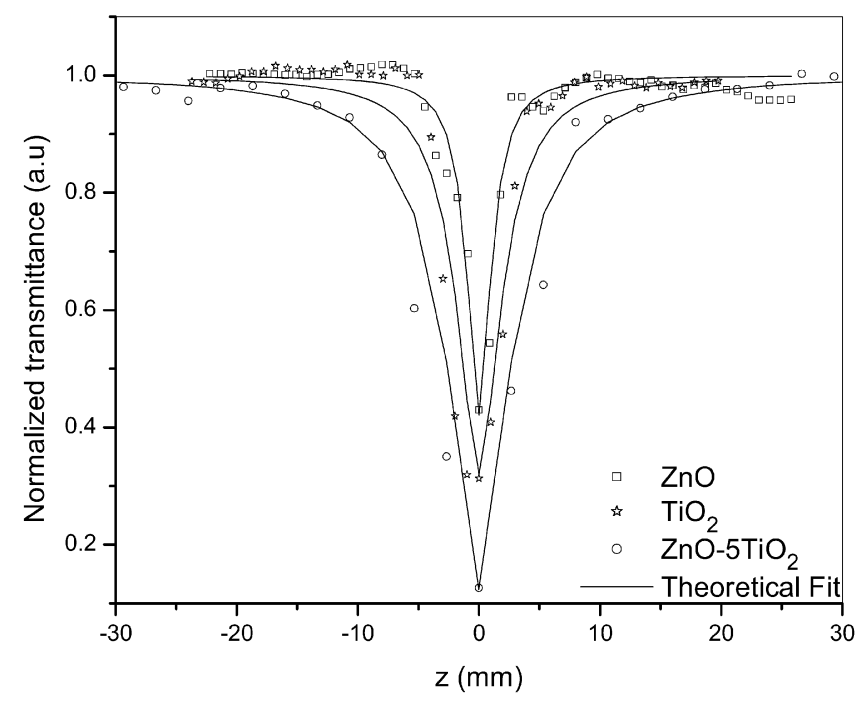

Fig. 4. Open aperture $z$ scan traces of $\mathrm{ZnO}-\mathrm{TiO}_{2}$ nanocomposites at an intensity of $300 \mathrm{MW} / \mathrm{cm}^{2}$ for an irradiation wavelength of $532 \mathrm{~nm}$. 
Table 1

Measured values of nonlinear absorption coefficient and refractive index of $\mathrm{ZnO}$ $\mathrm{TiO}_{2}$ nanocomposites at an intensity of $300 \mathrm{MW} / \mathrm{cm}^{2}$ for an irradiation wavelength of $532 \mathrm{~nm}$

\begin{tabular}{lcc}
\hline $\begin{array}{l}\mathrm{ZnO}-\mathrm{TiO}_{2} \\
\text { nanocomposites }\end{array}$ & $\begin{array}{l}\beta \\
(\mathrm{cm} / \mathrm{GW})\end{array}$ & $\begin{array}{l}n_{2} \\
\left(10^{-17} \mathrm{~m}^{2} / \mathrm{W}\right)\end{array}$ \\
\hline $\mathrm{ZnO}$ & 20.7 & 1.5 \\
$\mathrm{TiO}_{2}$ & 77.8 & 2.9 \\
$\mathrm{ZnO}-0.1 \mathrm{TiO}_{2}$ & 86.4 & 4.1 \\
$\mathrm{ZnO}-0.5 \mathrm{TiO}_{2}$ & 100.2 & 6.0 \\
$\mathrm{ZnO}-1 \mathrm{TiO}_{2}$ & 121.0 & 6.9 \\
$\mathrm{ZnO}-2 \mathrm{TiO}_{2}$ & 138.2 & 8.9 \\
$\mathrm{ZnO}-5 \mathrm{TiO}_{2}$ & 180.0 & 10.3
\end{tabular}

be of the third order, as it fits to a two photon absorption (TPA) process. The corresponding net transmission is given by [19]

$$
T(z)=\frac{C}{q_{0} \sqrt{\pi}} \int_{-\infty}^{\infty} \ln \left(1+q_{0} e^{-t^{2}}\right) d t,
$$

where $q_{0}(z, r, t)=\beta I_{0}(t) L_{\mathrm{eff}}$.

Here, $L_{\text {eff }}=1-e^{-\alpha l} / \alpha$ is the effective thickness with linear absorption coefficient $\alpha$, nonlinear absorption coefficient $\beta$ and $I_{0}$ is the irradiance at focus. The solid curves in Fig. 4 are the theoretical fit to the experimental data. The obtained values of nonlinear absorption coefficient $\beta$ at an intensity of $300 \mathrm{MW} / \mathrm{cm}^{2}$ are shown in Table 1.

Interestingly, $\mathrm{ZnO}$ and $\mathrm{TiO}_{2}$ colloids show a minimum nonlinearity, while the $\mathrm{ZnO}-\mathrm{TiO}_{2}$ nanocomposites clearly exhibit a larger induced absorption behavior. The calculated nonlinear coefficients given in Table 1 show fairly high values of nonlinearity. The nonlinear absorption coefficient increases substantially in the nanocomposites, as compared to pure $\mathrm{ZnO}$ and $\mathrm{TiO}_{2}$ colloids. The enhancement of the third-order nonlinearity can be attributed to the concentration of exciton oscillator strength [3].

Different processes, like two photon absorption, free carrier absorption, transient absorption, interband absorption, photoejection of electrons and nonlinear scattering are reported to be operative in nanoclusters. In general, induced absorption can occur due to a variety of processes. The theory of two photon absorption process fitted well with the experimental curve establishes that TPA is the basic mechanism. There is the possibility of higher order nonlinear processes such as free carrier absorption (FCA) contributing to induced absorption. The free carrier lifetime of $\mathrm{ZnO}$ is reported to be $2.8 \mathrm{~ns}$ [24]. Hence the $7 \mathrm{~ns}$ pulses used in the present study can excite the accumulated free carriers generated by TPA by the rising edge of the pulse. But the free carrier absorption is weak compared to TPA and hence the corresponding contribution in the $z$-scan curves is relatively less. The large optical nonlinearities in the nanosized particles containing $\mathrm{TiO}_{2}$ are reported to be due to TPA and two photon resonant exciton [25]. Thus we propose that the observed nonlinearity is caused by two photon absorption followed by free carrier absorption in the nanocomposites.

Fig. 5 gives the closed aperture $z$ scan traces of $\mathrm{ZnO}-\mathrm{TiO}_{2}$ nanocomposites at a fluence of $300 \mathrm{MW} / \mathrm{cm}^{2}$. The closed-aperture curve exhibits a peak-valley shape, indicating a negative value of the nonlinear refractive index $n_{2}$ [26]. For samples with sizeable refractive and absorptive nonlinearities, closed-aperture measurements contain contributions from both the intensity-dependent changes in the transmission and in refractive index [19]. By dividing the normalized closed-aperture transmittance by the corresponding normalized open-aperture data we can retrieve the phase distortion created due to the change in refractive index.
It is observed that the peak-valley of closed-aperture $z$ scan satisfied the condition $\Delta z \sim 1.7 z_{0}$, thus confirming the presence of pure electronic third order nonlinearity [19]. The value of the difference between the normalised peak and valley transmittance, $\Delta T_{\mathrm{p}-\mathrm{v}}$ can be obtained by the best theoretical fit from the results of divided $z$ scan curve. The nonlinear refractive index $n_{2}$ is calculated from $\Delta T_{\mathrm{p}-\mathrm{v}}$ in closed aperture $z$ scan using Eq. (2) and is tabulated in Table 1 ,

$$
\Delta T_{\mathrm{p}-\mathrm{v}}=0.406(1-S)^{0.25}\left|\Delta \Phi_{0}\right|, \quad \text { where }\left|\Delta \Phi_{0}\right|=\frac{2 \pi}{\lambda} n_{2} I_{0} L_{\mathrm{eff}}
$$

The peak-valley trace in a closed aperture $z$ scan shows that these samples have self-defocusing (negative, $n_{2}<0$ ) nonlinearity, though earlier reports with $780 \mathrm{~nm}$ picosecond pulsed lasers have shown positive nonlinearity for $\mathrm{PMMA}^{-\mathrm{TiO}_{2}}$ nanocomposites [27]. The nonlinear refractive index increases substantially in the nanocomposites, as compared to pure $\mathrm{ZnO}$ and $\mathrm{TiO}_{2}$ colloids. The enhancement of the third-order nonlinearity can be attributed to the concentration of exciton oscillator strength [3]. Since $n_{2}$ increases with absorption, thermal nonlinearity is also taken into account. It is reported that if the thermal contributions are to dominate, then there will be increase in $n_{2}$ with increase of absorption [28].

The significant optical nonlinearities of the pure semiconductor nanocolloid at $532 \mathrm{~nm}$ are reported to have the nonlinear refractive index of the order of $10^{-16}$ to $10^{-17} \mathrm{~m}^{2} / \mathrm{W}$. The third order optical nonlinearity, $n_{2}$ of $\mathrm{TiO}_{2}$ nanocrystalline particles dispersed in $\mathrm{SiO}_{2}$ is found to be $10^{-12}$ esu at $532 \mathrm{~nm}$ with nanosecond laser pulses [29]. It is worth noting that certain representative thirdorder nonlinear optical materials, such as $\mathrm{CuO}$ chain compounds, $\mathrm{Ag}_{2} \mathrm{~S}-\mathrm{CdS}$ nanocomposites, organic coated quantum dots, metal clusters, etc., yielded values of order of $10^{-9}$ to $10^{-14} \mathrm{~m} / \mathrm{W}$ for nonlinear absorption coefficient at a wavelength of $532 \mathrm{~nm}[30,31]$. The two photon absorption coefficient of $\mathrm{TiO}_{2}$ nanocrystals is reported to be $14 \mathrm{~cm} / \mathrm{GW}$ at $532 \mathrm{~nm}$ and these values are comparable to the value of $\beta$ obtained for nanocomposites in the present investigation [29]. Thus, the nonlinear absorption coefficient and nonlinear refractive index measured by the $z$ scan technique reveals that the $\mathrm{ZnO}-\mathrm{TiO}_{2}$ nanocomposites investigated in the present study have good nonlinear optical response and could be chosen as ideal candidates with potential applications in nonlinear optics.

Recently, nanomaterials have drawn significant attention as optical limiters for eyes or for sensor protection from laser terror in homeland or agile laser threats on the battlefield [32]. Also, the nonlinear optical properties of nanomaterials are of great interest for optical switching, pulse power shaping of OPO (optical parametric oscillator)/OPG (optical parametric generator), and other nonlinear optical applications. Optical power limiting is operated through the nonlinear optical processes of nanomaterials. However, the great potentials of nanomaterials as optical power limiters have just begun to be recognized.

To examine the viability of $\mathrm{ZnO}-\mathrm{TiO}_{2}$ nanocomposites as optical limiters, the nonlinear transmission of the colloid is studied as a function of input fluence. An important term in the optical limiting measurement is the limiting threshold. It is obvious that the lower the optical limiting threshold, the better the optical limiting material. Optical limiters are devices that transmit light at low input fluences or intensities, but become opaque at high inputs. The optical limiting property occurs mostly due to absorptive nonlinearity which corresponds to the imaginary part of third order susceptibility [33]. From the value of fluence at focus, the fluence values at other positions could be calculated using the standard equations for Gaussian beam waist. Such plots represent a better comparison of the nonlinear absorption or transmission in these samples and are generated from $z$ scan traces. Fig. 6 illustrates the 


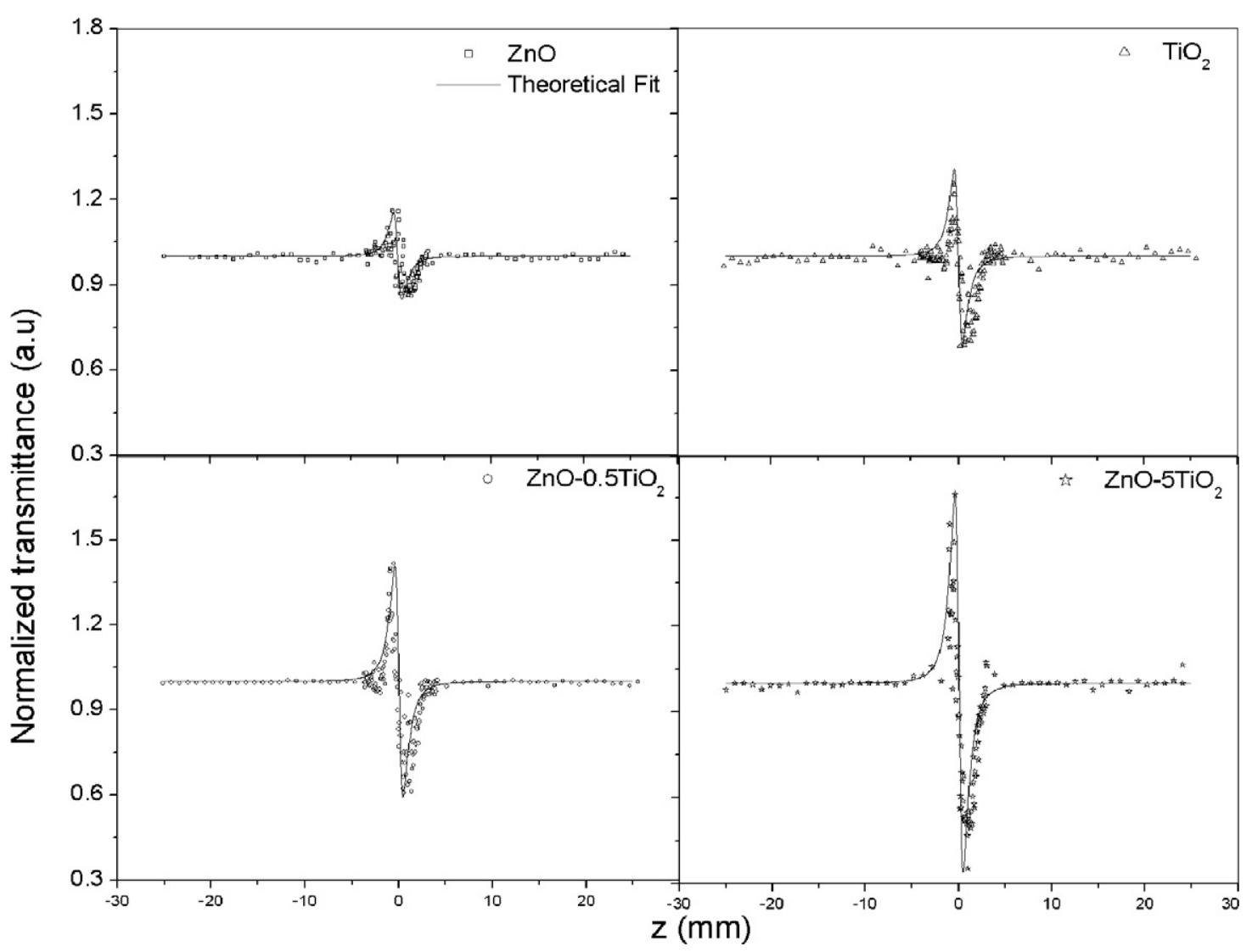

Fig. 5. Closed aperture $z$ scan traces of $\mathrm{ZnO}-\mathrm{TiO}_{2}$ nanocomposites at an intensity of $300 \mathrm{MW} / \mathrm{cm}^{2}$ for an irradiation wavelength of 532 nm.

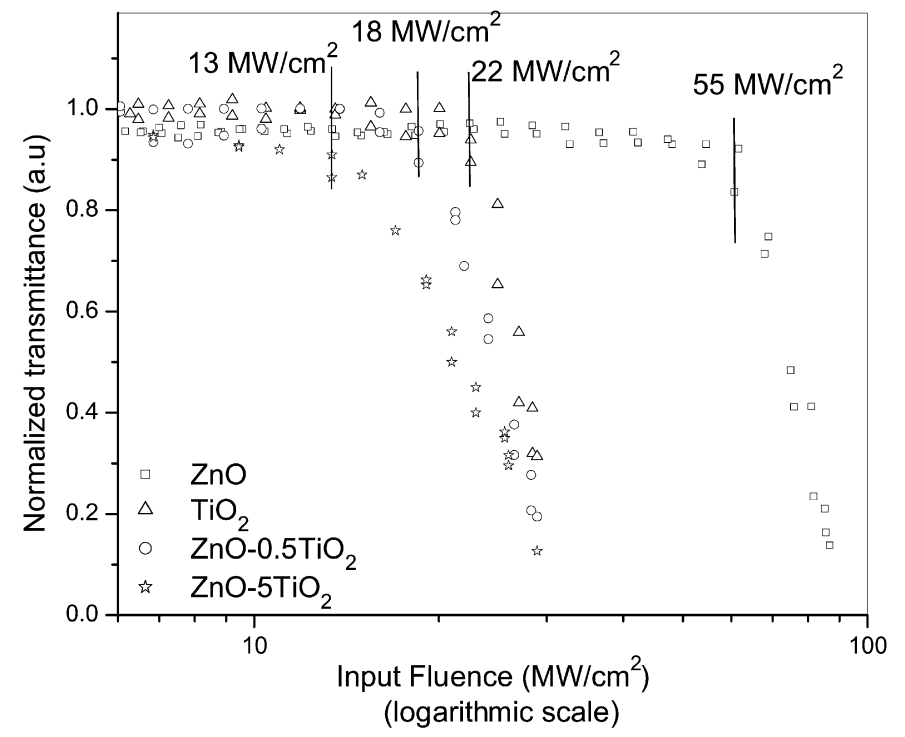

Fig. 6. Optical limiting response of $\mathrm{ZnO}-\mathrm{TiO}_{2}$ nanocomposites at $532 \mathrm{~nm}$.

influence of volume fraction of $\mathrm{TiO}_{2}$ in $\mathrm{ZnO}-\mathrm{TiO}_{2}$ nanocomposites on the optical limiting response.

The fluence value corresponding to the onset of optical limiting (optical limiting threshold) is found to be high in the case of $\mathrm{ZnO}$ colloids (55 MW/ $\mathrm{cm}^{2}$ ) in comparison to the $\mathrm{TiO}_{2}$ colloids $\left(22 \mathrm{MW} / \mathrm{cm}^{2}\right)$. These values are of the order of the reported optical

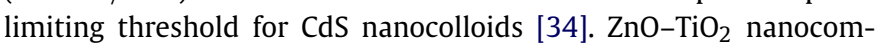
posites are found to be good optical limiters compared to $\mathrm{ZnO}$ and $\mathrm{TiO}_{2}$ and the optical limiting threshold of $\mathrm{ZnO}-5 \mathrm{TiO}_{2}$ nanocomposites is observed to be $13 \mathrm{MW} / \mathrm{cm}^{2}$. The arrow in the figure indicates the approximate fluence at which the normalized transmission begins to deviate from linearity. Nanocomposites have a significant effect on the limiting performance and increasing the volume fraction of $\mathrm{TiO}_{2}$ reduces the limiting threshold and enhances the optical limiting performance.

\section{Conclusion}

The spectral and nonlinear optical properties of $\mathrm{ZnO}-\mathrm{TiO}_{2}$ nanocomposites prepared by a colloidal chemical synthesis are investigated. The optical band gap is tunable between $3.84 \mathrm{eV}$ and $4.12 \mathrm{eV}$. Emission peaks of $\mathrm{ZnO}-\mathrm{TiO}_{2}$ nanocomposites changes from $340 \mathrm{~nm}$ to $385 \mathrm{~nm}$ almost in proportion to changes in $E_{\mathrm{g}}$. It is possible to obtain a desired wavelength of UV luminescence by simply adjusting the composition. Nonlinear optical response of these samples is studied using nanosecond laser pulses at $532 \mathrm{~nm}$. The $\mathrm{ZnO}-\mathrm{TiO}_{2}$ nanocomposites show self-defocusing nonlinearity and good nonlinear absorption behaviour. The observed nonlinear absorption is explained through two photon absorption followed by free carrier absorption. The nonlinear refractive index and the nonlinear absorption increases with increasing $\mathrm{TiO}_{2}$ volume fraction at $532 \mathrm{~nm}$ and can be attributed to the enhancement of exciton oscillator strength. It is shown that $\mathrm{ZnO}^{-\mathrm{TiO}_{2}}$ is a potential nanocomposite material for tunable light emission and for the development of nonlinear optical devices with a relatively small limiting threshold.

\section{Acknowledgment}

L.I. acknowledges UGC for research fellowship.

\section{References}

[1] Y. Kayanuma, Phys. Rev. B 38 (1988) 9797. 
[2] Z.L. Wang, Mater. Today 7 (2004) 26.

[3] A.P. Alivisatos, J. Phys. Chem. 100 (1996) 13226.

[4] N. Suzuki, Y. Tomita, T. Kojima, Appl. Phys. Lett. 81 (2002) 4121.

[5] S.X. Wang, L.D. Zhang, H. Su, Z.P. Zhang, G.H. Li, G.W. Meng, J. Zhang, Y.W. Wang, J.C. Fan, T. Gao, Phys. Lett. A 281 (2001) 59.

[6] U. Kreibig, M. Vollmer, Optical Properties of Metal Clusters, Springer, Berlin, 1995.

[7] B. Kraeutler, A.J. Bard, J. Am. Chem. Soc. 100 (1978) 4317.

[8] A.E. Hichou, A. Bougrine, J.L. Bubendorff, J. Ebothe, M. Addou, M. Troyon, Semicond. Sci. Technol. 17 (2002) 607.

[9] X.H. Wang, J.L. Shi, S.G. Dai, Y. Yang, Thin Solid Films 429 (2003) 102.

[10] P. Yang, C.F. Song, M.K. Lu, X. Yin, G.J. Zhou, D. Xu, D.R. Yuan, Chem. Phys. Lett. 345 (2001) 429.

[11] M.Y. Han, W. Huang, C.H. Chew, L.M. Gan, X.J. Zhang, W. Ji, J. Phys. Chem. B 102 (1998) 1884.

[12] A. Nakamura, Y.L. Lee, T. Kataoka, T. Tokizaki, J. Lumin. 60-61 (1994) 376.

[13] W.S. Shi, O. Agyeman, C.N. Xu, J. Appl. Phys. 91 (2002) 5640.

[14] D.M. Bagnall, Y.F. Chen, Z. Zhu, T. Yao, S. Koyama, M.Y. Shen, T. Goto, Appl. Phys. Lett. 70 (1997) 2230.

[15] Ü. Özgür, Ya.I. Alivov, C. Liu, A. Teke, M.A. Reshchikov, S. Doğan, V. Avrutin, S.-J. Cho, H. Morkoç, J. Appl. Phys. 98 (2005) 041301.

[16] A. Tsukazaki, A. Ohtomo, T. Onuma, M. Ohtani, T. Makino, M. Sumiya, K. Ohtani, S.F. Chichibu, S. Fuke, Y. Segawa, H. Ohno, H. Koinuma, M. Kawasaki, Nat. Mater. 4 (2005) 42.
[17] E.W. Seelig, B. Tang, A. Yamilov, H. Cao, R.P.H. Chang, Mater. Chem. Phys. 80 (2002) 257.

[18] R. Fernandes de Farias, J. Colloid Interface Sci. 239 (2001) 584.

[19] M.S. Bahae, A.A. Said, E.W. van Stryland, Opt. Lett. 14 (1989) 955.

[20] R. Viswanatha, S. Sapra, B. Satpati, P.V. Satyam, B.N. Dev, D.D. Sharma, J. Mater Chem. 14 (2004) 661.

[21] J. Piprek, Semiconductor Optoelectronic Devices: Introduction to Physics and Simulation, Academic Press, San Diego, 2003.

[22] V.L. Colvin, A.P. Alivisatos, J.G. Tobin, Phys. Rev. Lett. 66 (1991) 2786.

[23] S. Shionoya, W.M. Yen, Phosphor Handbook, CRC Press, New York, 1998.

[24] X.J. Zhang, W. Ji, S.H. Tang, J. Opt. Soc. Am. B 14 (1997) 1951.

[25] A. Penzkofer, W. Falkenstein, Opt. Commun. 17 (1976) 1.

[26] H.S. Waff, K. Park, Phys. Lett. A 32 (1970) 109.

[27] H.I. Elim, W. Ji, A.H. Yuwono, J.M. Xue, J. Wang, Appl. Phys. Lett. 82 (2003) 2691.

[28] P. Prem Kiran, G. De, D. Narayana Rao, IEE Proc. Circuits Devices Syst. 150 (2003) 559.

[29] Y. Watanabe, M. Ohnishi, T. Tsuchiya, Appl. Phys. Lett. 66 (1995) 3431.

[30] M.Y. Han, W. Huang, C.H. Chew, L.M. Gan, X.J. Zhang, W. Ji, J. Phys. Chem. B 102 (1998) 1884.

[31] S. Shi, W. Ji, S.H. Tang, J. Am. Chem. Soc. 116 (1994) 3615.

[32] Y. Sun, J.E. Riggs, K.B. Henbest, R.B. Martin, J. Nonlinear Opt. Phys. Mater. 9 (2000) 481.

[33] F.M. Quereshi, S.J. Martin, X. Long, D.D.C. Bradley, F.Z. Heneri, W.J. Balu, E.C. Smith, C.H. Wang, A.K. Kar, H.L. Anderson, Chem. Phys. 231 (1998) 87.

[34] W. Jia, E.P. Douglas, F. Guo, W. Suna, Appl. Phys. Lett. 85 (2004) 6326. 